\title{
Hierarchical Segmentation of Polarimetric SAR Images Using Heterogeneous Clutter Models
}

\author{
Lionel Bombrun, Member, IEEE, Gabriel Vasile, Member, IEEE, Michel Gay, Member, IEEE, and Felix Totir
}

\begin{abstract}
In this paper, heterogeneous clutter models are used to describe polarimetric synthetic aperture radar (PolSAR) data. The KummerU distribution is introduced to model the PolSAR clutter. Then, a detailed analysis is carried out to evaluate the potential of this new multivariate distribution. It is implemented in a hierarchical maximum likelihood segmentation algorithm. The segmentation results are shown on both synthetic and highresolution PoISAR data at the $\mathrm{X}$ - and L-bands. Finally, some methods are examined to determine automatically the "optimal" number of segments in the final partition.
\end{abstract}

Index Terms-Fisher probability density function (PDF), KummerU PDF, polarimetric synthetic aperture radar (PolSAR) data, segmentation, spherically invariant random vectors (SIRV).

\section{INTRODUCTION}

B ECAUSE of its all-weather and all-day monitoring capabilities, synthetic aperture radar (SAR) imagery has been widely used for global Earth monitoring. Such systems offer a number of advantages for Earth-surface and feature observation compared to optical sensors. With the new generation of highresolution SAR sensors, the high-quality images of the Earth's surface are acquired. They offer the opportunity to observe thinner spatial features from space. Nevertheless, with such sensors, only a small number of scatterers are present in each resolution cell. The xlassical statistical models can therefore be reconsidered. Many works have been recently dedicated to this problem. For example, Delignon et al. [1] have proposed to use the Pearson system KUBW for the statistical modeling of ocean SAR images. Other works have proposed to model agricultural fields and urban areas in high-resolution SAR images by means of the Fisher probability density functions (PDFs) [2], [3].

Polarimetric SAR (PolSAR) data describe the interactions between the electromagnetic wave and the scatterers present in each resolution cell. By means of the Sinclair matrix, the polarimetric diversity has been widely studied to retrieve the physical properties of the media [4]-[6]. For low-resolution images, the classical Wishart distribution has been used in the classification and segmentation of PolSAR data [7], [8]. With

Manuscript received November 20, 2009; revised April 29, 2010.

L. Bombrun is with the Grenoble Image sPeech Signal Automatics Laboratory (GIPSA-Lab), Grenoble Institute of Technology, 38402 Grenoble, France, and also with the Supelec, ONERA, NUS, DSTA Research Alliance (SONDRA), 91192 Gif-sur-Yvette, France (e-mail: lionel.bombrun@gipsalab.grenoble-inp.fr).

G. Vasile, M. Gay, and F. Totir are with the GIPSA-Lab, Grenoble Institute of Technology, 38402 Grenoble, France (e-mail: gabriel.vasile@gipsa-lab. grenoble-inp.fr; michel.gay@gipsa-lab.grenoble-inp.fr; felix.totir@gipsa-lab. grenoble-inp.fr).

Color versions of one or more of the figures in this paper are available online at http://ieeexplore.ieee.org.

Digital Object Identifier 10.1109/TGRS.2010.2060730 the new PolSAR sensors, the number of scatterers present in each resolution cell decreases considerably. The homogeneous hypothesis of the PolSAR clutter can be reconsidered. To overcome this difficulty, Anfinsen et al. [9] have introduced the relaxed Wishart distribution, which has shown promising results in modeling the forested scenes. Other heterogeneous clutter models have been proposed in the literature by means of the scalar product model. In this model, the spatial nonhomogeneity is incorporated by modeling the clutter as the product between the square root of a scalar random variable (texture) and an independent zero-mean complex circular Gaussian random vector (speckle). If the texture random variable is Gamma distributed, the target scattering vector follows the well-known $\mathcal{K}$ distribution [10]-[12]. Another class of multivariate distribution has been introduced : the $\mathcal{G}^{0}$ polarimetric distribution [13]. It assumes an inverse Gamma distributed texture. This model is able to fit an extremely heterogeneous clutter compared to the $\mathcal{K}$ distribution. Recently, some works have proposed to generalize those two classes with the multivariate KummerU distribution which implies a Fisher distributed texture [14], [15].

This paper is organized as follows. In Section II, the multivariate KummerU distribution is introduced. Some benefits of this model are discussed. Then, in Section III, the KummerU is implemented in a hierarchical maximum-likelihood (ML) segmentation algorithm. The segmentation results are next analyzed in both synthetic and high-resolution PolSAR images. Section IV presents some results to determine automatically the number of segments in the final partition. Finally, some conclusion and perspectives of this paper are discussed in Section V.

\section{KummerU Heterogeneous Clutter}

With the new generation of airborne and spaceborne SAR sensors, the number of scatterers present in each resolution cell decreases considerably. In certain applications such as segmentation or detection, the central limit theorem cannot be directly used to support the Gaussianity hypothesis of the backscattered clutter. The homogeneous hypothesis of the PolSAR clutter must then be reconsidered. Heterogeneous clutter models have therefore recently been studied. The generalizations of the Gaussian distribution are appealing since they allow to retrieve the basic Gaussian model under particular assumptions. Spherically invariant random processes (SIRPs) or vectors (SIRVs) are such a generalization. The SIRV has first been introduced by Yao [16] for the estimation/detection in the information theory. In the PolSAR, the target vector $\mathbf{k}$ is a complex vector of length three or four and could be written under the SIRV model hypothesis [17]. It is defined as the product of a square root 
This article has been accepted for inclusion in a future issue of this journal. Content is final as presented, with the exception of pagination.

TABLE I

KUMMERU PDF AND PARTiCUlar CASES

\begin{tabular}{|c|c|c|c|}
\hline \multicolumn{2}{|r|}{ Texture PDF } & \multicolumn{2}{|r|}{ SIRV PDF } \\
\hline Name & $p_{\tau}(\tau)$ & Name & $p_{\mathbf{k}}(\mathbf{k})$ \\
\hline Dirac & $\delta(\tau-1)$ & Gaussian & $\frac{1}{\pi^{p}|[M]|} \exp \left(-\mathbf{k}^{H}[M]^{-1} \mathbf{k}\right)$ \\
\hline Gamma & $\frac{1}{\Gamma(\mathcal{L})} \frac{\mathcal{L}}{m}\left(\frac{\mathcal{L} \tau}{m}\right)^{\mathcal{L}-1} e^{-\frac{\mathcal{L} \tau}{m}}$ & $\mathcal{K}$ & $\frac{2}{\pi^{p} \Gamma(\mathcal{L})|[M]|}\left(\frac{\mathcal{L}}{m}\right)^{\left(\frac{\mathcal{L}+p}{2}\right)}\left[\mathbf{k}^{H}[M]^{-1} \mathbf{k}\right]^{\left(\frac{\mathcal{L}-p}{2}\right)}$ BesselK $_{p-\mathcal{L}}\left(2 \sqrt{\frac{\mathcal{L} \mathbf{k}^{H}[M]^{-1} \mathbf{k}}{m}}\right)$ \\
\hline $\begin{array}{l}\text { Inverse } \\
\text { Gamma }\end{array}$ & $\frac{1}{\Gamma(\mathcal{M})} \frac{1}{\mathcal{M} m}\left(\frac{\mathcal{M} m}{\tau}\right)^{\mathcal{M}+1} e^{-\frac{\mathcal{M} m}{\tau}}$ & $\mathcal{G}^{0}$ & $\frac{1}{\pi^{p}|[M]|} \frac{(m \mathcal{M})^{\mathcal{M}}}{\Gamma(\mathcal{M})} \Gamma(p+\mathcal{M})\left(\mathbf{k}^{H}[M]^{-1} \mathbf{k}+m \mathcal{M}\right)^{-(\mathcal{M}+p)}$ \\
\hline Fisher & $\frac{\Gamma(\mathcal{L}+\mathcal{M})}{\Gamma(\mathcal{L}) \Gamma(\mathcal{M})} \frac{\mathcal{L}}{\mathcal{M} m} \frac{\left(\frac{\mathcal{L} \tau}{\mathcal{M} m}\right)^{\mathcal{L}-1}}{\left(1+\frac{\mathcal{L} \tau}{\mathcal{M} m}\right)^{\mathcal{L}+\mathcal{M}}}$ & KummerU & $\frac{1}{\pi^{p}|[M]|} \frac{\Gamma(\mathcal{L}+\mathcal{M})}{\Gamma(\mathcal{L}) \Gamma(\mathcal{M})}\left(\frac{\mathcal{L}}{\mathcal{M} m}\right)^{p} \Gamma(p+\mathcal{M}) U\left(p+\mathcal{M} ; 1+p-\mathcal{L} ; \frac{\mathcal{L}}{\mathcal{M} m} \mathbf{k}^{H}[M]^{-1} \mathbf{k}\right)$ \\
\hline
\end{tabular}

of a positive random variable $\tau$ (representing the texture) with an independent circular complex Gaussian vector $\mathbf{z}$ with zero mean and covariance matrix $[M]=E\left\{\mathbf{z z}^{H}\right\}$ (representing the speckle)

$$
\mathbf{k}=\sqrt{\tau} \mathbf{z}
$$

where the superscript $H$ denotes the complex conjugate transposition and $E\{\cdot\}$ denotes the mathematical expectation.

The SIRV representation is not unique, so a normalization condition is necessary. Indeed, if $\left[M_{1}\right]$ and $\left[M_{2}\right]$ are two covariance matrices such that $\left[M_{1}\right]=\alpha\left[M_{2}\right]$ with $\alpha \in \mathbb{R}^{+*}$, then $\left\{\tau_{1},\left[M_{1}\right]\right\}$ and $\left\{\tau_{2}=\tau_{1} / \alpha,\left[M_{2}\right]\right\}$ describe the same SIRV. In this paper, the trace of the covariance matrix is normalized to $p$, the dimension of the target scattering vector $(p=3$ for the reciprocal case) [17], [18].

For a given covariance matrix $[M]$, the ML estimator of the texture for the pixel $i\left(\tau_{i}\right)$ is given by

$$
\hat{\tau}_{i}=\frac{\mathbf{k}_{i}^{H}[M]^{-1} \mathbf{k}_{i}}{p} .
$$

The ML estimator of the normalized covariance matrix under the deterministic texture case is the solution of the following recursive equation

$$
\begin{aligned}
& {[\hat{M}]_{F P}=f\left([\hat{M}]_{F P}\right)=\frac{p}{N} \sum_{i=1}^{N} } \frac{\mathbf{k}_{i} \mathbf{k}_{i}^{H}}{\mathbf{k}_{i}^{H}[\hat{M}]_{F P}^{-1} \mathbf{k}_{i}}, \\
& \text { with } \operatorname{Tr}\left([\hat{M}]_{F P}\right)=p .
\end{aligned}
$$

Pascal et al. have established the existence and the uniqueness, up to a scalar factor, of the fixed point estimator of the normalized covariance matrix, as well as the convergence of the recursive algorithm, whatever the initialization [18], [19].

When the texture is assumed to be deterministic, the ML estimator of the normalized covariance matrix is given by $\hat{M}_{F P}$ in (3). However, when the texture is a random variable, $\hat{M}_{F P}$ is not the ML estimator, it is an "approximate" ML estimator. The ML estimator of the normalized covariance matrix depends on the texture PDF $p_{\tau}(\tau)$, and its expression is linked with the density generator function $h_{p}(x)$ by

$$
\left[\hat{M}_{M L}\right]=\frac{1}{N} \sum_{i=1}^{N} \frac{h_{p+1}\left(\mathbf{k}_{i}^{H}\left[\hat{M}_{M L}\right]^{-1} \mathbf{k}_{i}\right)}{h_{p}\left(\mathbf{k}_{i}^{H}\left[\hat{M}_{M L}\right]^{-1} \mathbf{k}_{i}\right)} \mathbf{k}_{i} \mathbf{k}_{i}^{H}
$$

where the expression of the density generator function is given by [20], [21]

$$
h_{p}(x)=\int_{0}^{+\infty} \frac{1}{\tau^{p}} \exp \left(-\frac{x}{\tau}\right) p_{\tau}(\tau) d \tau .
$$

Chitour and Pascal have proven that (4) admits a unique solution and that its corresponding iterative algorithm converges to the fixed point solution for every admissible initial condition [22].

When the texture is a random variable with a given PDF, the expression of the density generator function $h_{p}(\cdot)$ given by (5) can be computed. If $h_{p}(\cdot)$ has an analytical expression, the ML estimator can be computed according to (4). When the texture is a random variable with an unknown PDF, the density generator function cannot be computed numerically. The ML estimator $\hat{M}_{M L}$ cannot be found, and the approximate ML estimator $\hat{M}_{F P}$ should be used instead.

It is important to notice that, in the SIRV definition, the PDF of the texture random variable is not explicitly specified. As a consequence, the SIRVs describe a whole class of stochastic processes. This class includes the conventional clutter models having Gaussian, $\mathcal{K}$-distributed, Rayleigh, or Weibull PDFs. Some of those distributions are shown in Table I.

According to the SIRV estimation scheme, the scalar texture parameter can be extracted from a PolSAR data set [17]. In the next sections, the analysis of this parameter is carried out, and a statistical model is proposed. Then, a new target scattering PDF is derived, and some properties are exposed.

\section{A. Scalar Texture Modeling}

1) Fisher PDF: Fisher PDFs are known as the type VI solution of the Pearson system. They are introduced as the Mellin convolution (denoted as $\hat{\star}$ ) of a Gamma PDF by an inverse Gamma PDF. Their PDF is defined by three parameters as [23]

$$
\begin{aligned}
\mathcal{F}[\tau \mid m, \mathcal{L}, \mathcal{M}] & =\mathcal{G}[m, \mathcal{L}] \hat{\star} \mathcal{G}[1, \mathcal{M}] \\
& =\frac{\Gamma(\mathcal{L}+\mathcal{M})}{\Gamma(\mathcal{L}) \Gamma(\mathcal{M})} \frac{\mathcal{L}}{\mathcal{M} m} \frac{\left(\frac{\mathcal{L} \tau}{\mathcal{M} m}\right)^{\mathcal{L}-1}}{\left(1+\frac{\mathcal{L} \tau}{\mathcal{M} m}\right)^{\mathcal{L}+\mathcal{M}}}
\end{aligned}
$$

with $\mathcal{L}>0$ and $\mathcal{M}>0$. $m$ is a shape parameter. $\mathcal{L}$ and $\mathcal{M}$ are two shape parameters which control the behavior of the Fisher PDF between the heavy head and heavy tail distributions. The low values of the shape parameters lead to a significant texture. 


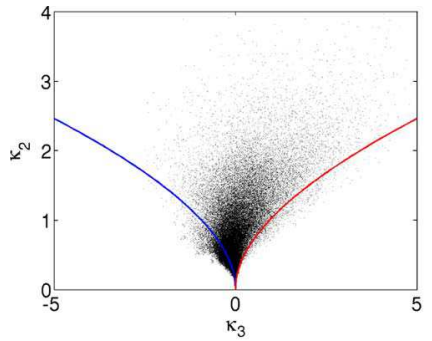

(a)

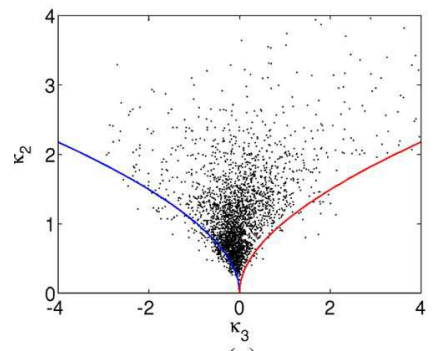

(c)

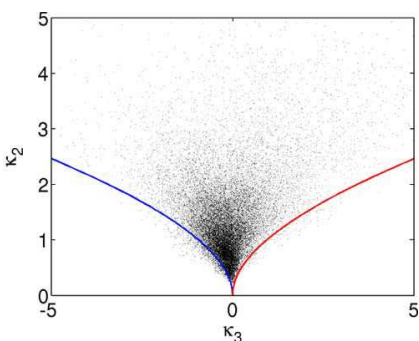

(b)

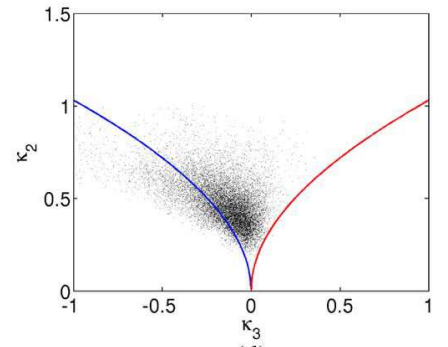

(d)
Fig. 1. $\kappa_{2} / \kappa_{3}$ plan for different bands and test sites. (a) X-band, Radar Aéroporté Multi-Spectral d'Etude des Signatures (RAMSES), Brétigny, France. (b) C-band, Convair, Ottawa, Canada. (c) L-band, Experimental SAR (ESAR), Oberpfaffenhofen, Germany. (d) P-band, RAMSES, Nezer, France.

2) Fisher PDF Parameter Estimation: Recent works have proposed to estimate the parameters of the Fisher PDF with the log-cumulants method, defined in the special framework of second kind statistics (where statistics-such as the second kind characteristic function - are defined using the Mellin transform instead of the Fourier transform) [23]. Nevertheless, these estimators are not ML estimators. The latter benefits from very desirable mathematical properties (asymptotic efficiency) and will be used through this paper. A description of the ML Fisher parameter estimation can be found in Appendix B.

3) Benefit of Fisher PDF: To evaluate the potential and limits of the Fisher PDFs to model the texture of PolSAR images, four data sets at the $\mathrm{X}-, \mathrm{C}-, \mathrm{L}-$, and P-bands are analyzed. From those data sets, the covariance matrix $[M]_{F P}$ and texture parameter $\tau$ are estimated on a sliding $7 \times 7$ window according to (2) and (3). Then, the second $\left(\kappa_{2}\right)$ and third $\left(\kappa_{3}\right)$ log-cumulants of the texture parameter are computed. Next, the $\kappa_{2} / \kappa_{3}$ plan is plotted. In this plan, the Gamma and inverse Gamma PDFs are represented by the blue and red lines, respectively. The Fisher PDFs cover all the space between the blue and red lines [2], [24]. Fig. 1 shows the $\kappa_{2} / \kappa_{3}$ plan for the different bands and test sites (fields, forest, urban area, etc.). It shows that the Fisher PDFs can be suitable to model the scalar texture parameter of PolSAR data.

Table II shows the percentage of points $p_{\text {not }}$ Fisher which are outside the Fisher PDF domain definition. This percentage can be divided into two categories: the points which belong to the Beta model, denoted by $p_{\text {Beta }}$, (i.e., the points located under the blue Gamma line) and those which satisfy the inverse Beta PDF, denoted by $p_{\text {Inverse Beta }}$, (i.e., the points located under the red inverse Gamma line). It yields that, for the four studied data sets, at least $75 \%$ of the data belong to the Fisher $\kappa_{2} / \kappa_{3}$ domain. The other amount of points which does not satisfy the Fisher model is due to both the estimation errors (log-cumulants $\kappa_{2}$ and $\kappa_{3}$ are computed on a sliding $7 \times 7$ square window) and
TABLE II

Percentage of Points Outside the FISHER PDFS DOMAIN DEFINITION

\begin{tabular}{||c|c|c|c|c||}
\hline \hline data set & $\begin{array}{c}\text { RAMSES } \\
\text { Brétigny } \\
\text { X-band }\end{array}$ & $\begin{array}{c}\text { CONVAIR } \\
\text { Ottawa } \\
\text { C-band }\end{array}$ & $\begin{array}{c}\text { ESAR } \\
\text { Oberpfaffenhofen } \\
\text { L-band }\end{array}$ & $\begin{array}{c}\text { RAMSES } \\
\text { Nezer } \\
\text { P-band }\end{array}$ \\
\hline$p_{\text {Beta }}$ & $16.72 \%$ & $3.61 \%$ & $6.31 \%$ & $24.35 \%$ \\
\hline$p_{\text {Inverse Beta }}$ & $1.35 \%$ & $0.63 \%$ & $1.23 \%$ & $0.06 \%$ \\
\hline$p_{\text {not Fisher }}$ & $18.07 \%$ & $4.24 \%$ & $7.54 \%$ & $24.41 \%$ \\
\hline \hline
\end{tabular}

real data properties. For the RAMSES P-band data set over the Nezer Forest [see Fig. 1(d)], a trend in the $\kappa_{2} / \kappa_{3}$ plan can be observed around the blue line (Gamma model).

\section{B. Target Scattering PDF}

For a given texture PDF $p_{\tau}(\tau)$, the expression of the target scattering vector PDF is given by

$$
\begin{aligned}
p_{\mathbf{k}}(\mathbf{k}) & =\frac{1}{\pi^{p}|[M]|} \int_{0}^{+\infty} \frac{1}{\tau^{p}} \exp \left(-\frac{\mathbf{k}^{H}[M]^{-1} \mathbf{k}}{\tau}\right) p_{\tau}(\tau) d \tau \\
& =\frac{1}{\pi^{p}|[M]|} h_{p}\left(\mathbf{k}^{H}[M]^{-1} \mathbf{k}\right) .
\end{aligned}
$$

1) KummerU PDF: For a Fisher distributed texture, the target scattering vector PDF has been mathematically established [14], [15]

$$
\begin{aligned}
p_{\mathbf{k}}(\mathbf{k} \mid[M], m, \mathcal{L}, \mathcal{M})=\frac{1}{\pi^{p}|[M]|} & \frac{\Gamma(\mathcal{L}+\mathcal{M})}{\Gamma(\mathcal{L}) \Gamma(\mathcal{M})}\left(\frac{\mathcal{L}}{\mathcal{M} m}\right)^{p} \\
& \times \Gamma(p+\mathcal{M}) U(a ; b ; z)
\end{aligned}
$$

with $\quad a=p+\mathcal{M}, \quad b=1+p-\mathcal{L}, \quad$ and $\quad z=$ $(\mathcal{L} / \mathcal{M} m) \mathbf{k}^{H}[M]^{-1} \mathbf{k} .|\cdot|$ and $U(\cdot ; \cdot ; \cdot)$ denote the determinant operator and the confluent hypergeometric function of the second kind (KummerU), respectively. Hereinafter, this multivariate distribution is named the KummerU PDF.

Consequently, the expression of the density generator function is given by

$h_{p}\left(\mathbf{k}^{H}[M]^{-1} \mathbf{k}\right)=\frac{\Gamma(\mathcal{L}+\mathcal{M})}{\Gamma(\mathcal{L}) \Gamma(\mathcal{M})}\left(\frac{\mathcal{L}}{\mathcal{M} m}\right)^{p} \Gamma(p+\mathcal{M}) U(a ; b ; z)$.

2) Asymptotic Cases: As Fisher distributions are a generalization of the Gamma and inverse Gamma PDFs, the asymptotic cases of the KummerU PDF can be studied.

1) For large $\mathcal{M}$, the Fisher PDFs have the same behavior as the Gamma PDFs. In Appendix A, a proof of the convergence of the KummerU PDF toward the wellknown $\mathcal{K}$ PDF is achieved.

2) A similar approach can be done if $\mathcal{L}$ tends toward infinity. The Fisher PDFs have, therefore, the same behavior as the inverse Gamma PDFs. It yields that the KummerU PDF tends toward the multivariate Student distribution, also known as the $\mathcal{G}^{0}$ PDF [9], [13].

3) For the high values of $\mathcal{L}$ and $\mathcal{M}$, the texture parameter becomes less and less significant. Consequently, for the high values of the shape parameters, the KummerU PDF tends toward the Gaussian distribution. 


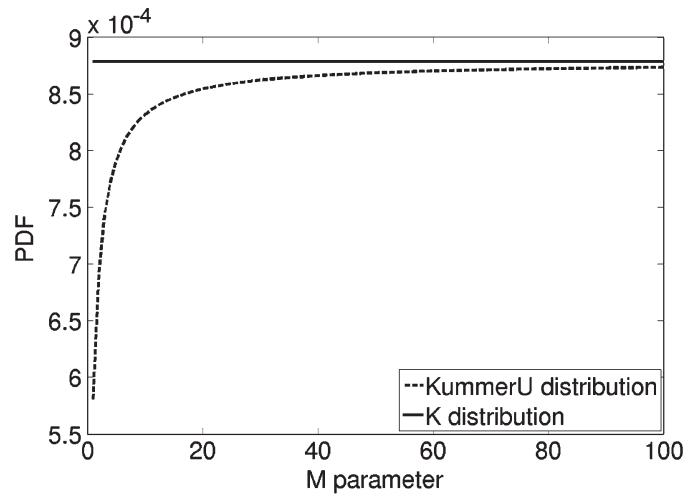

(a)

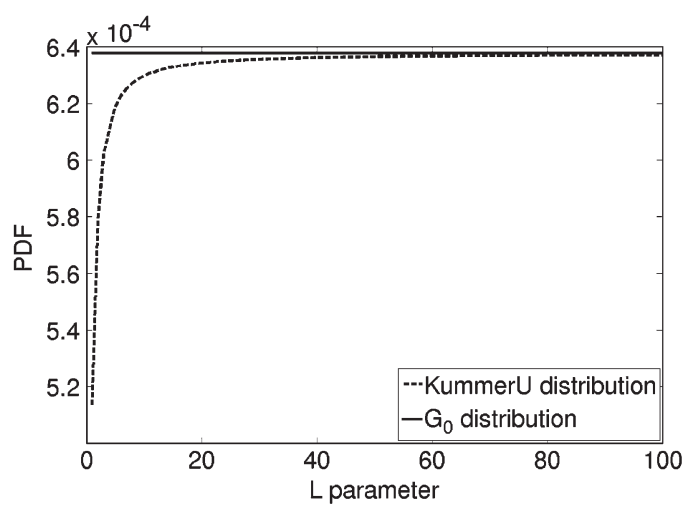

(b)

Fig. 2. Convergence of the KummerU PDF toward (a) the $\mathcal{K}$ distribution and (b) the $\mathcal{G}^{0}$ distribution as a function of the shape parameters $\mathcal{M}$ and $\mathcal{L}$.

Fig. 2 shows the convergence of the KummerU distribution toward the $\mathcal{K}$ and $\mathcal{G}^{0}$ distributions as a function of the shape parameters $\mathcal{M}$ and $\mathcal{L}$. The KummerU PDFs can be viewed as an extension of the classical multivariate $\mathcal{K}$ and $\mathcal{G}^{0}$ PolSAR models (see Table I for the analytical expressions).

\section{ML Parameter Estimation}

For a KummerU distributed clutter, one can replace in (4) the density generator function by its expression given in (9) to derive the expression of the ML estimator of the covariance matrix. It yields [15]

$$
\begin{aligned}
{\left[\hat{M}_{M L}\right]=} & \frac{p+\mathcal{M}}{N}\left(\frac{\mathcal{L}}{\mathcal{M} m}\right) \\
& \times \sum_{i=1}^{N} \frac{U\left(p+1+\mathcal{M} 2+p-\mathcal{L} \frac{\mathcal{L}}{\mathcal{M} m} \mathbf{k}_{i}^{H}\left[\hat{M}_{M L}\right]^{-1} \mathbf{k}_{i}\right)}{U\left(p+\mathcal{M} 1+p-\mathcal{L} \frac{\mathcal{L}}{\mathcal{M} m} \mathbf{k}_{i}^{H}\left[\hat{M}_{M L}\right]^{-1} \mathbf{k}_{i}\right)} \\
& \times \mathbf{k}_{i} \mathbf{k}_{i}^{H} .
\end{aligned}
$$

\section{HierarchicAl SEgmentation}

In this section, a segmentation application of the new multivariate KummerU PolSAR model is proposed. The hierarchical segmentation algorithm proposed by Beaulieu and Touzi [12] is adapted to the KummerU distributed target scattering vector. The segmentation algorithm is a classical iterative merge algorithm. At each iteration, the two four-connex segments (regions) which minimize the stepwise criterion (SC) are merged.

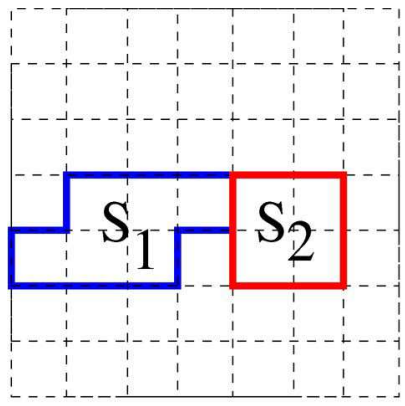

(a)

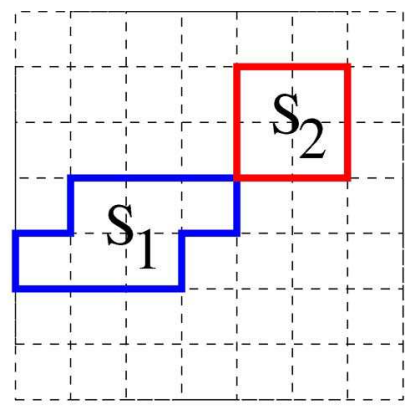

(b)
Fig. 3. (a) Four-connex segment pair. (b) Non-four-connex segment pair.

A four-connex segment pair is a group of two segments where at least one pixel of the first segment is in the neighborhood of one pixel of the second segment with the four-connexity sense. For illustrative purpose, a four-connex segment pair is shown in Fig. 3(a), and a non-four-connex segment pair is shown in Fig. 3(b).

The basic principle of the hierarchical segmentation algorithm can be divided into three steps.

1) Definition of an initial partition.

2) For each four-connex segment (regions) pair, the SC is computed. Then, the two segments which minimize the criterion are found and merged.

3) Stop if the maximum number of merges is reached; otherwise, go to Step 2.

\section{A. Similarity Measure}

At each iteration, merging two segments yields a decrease in the log-likelihood function. The SC is based on this consideration. The hierarchical segmentation algorithm merges the two adjacent segments $S_{i}$ and $S_{j}$ which minimizes the loss of likelihood of the partition (which is defined as the sum of likelihoods of the partition's segments). The $\mathrm{SC}\left(\mathrm{SC}_{i, j}\right)$ can be expressed as [12]

$$
\mathrm{SC}_{i, j}=\operatorname{MLL}\left(S_{i}\right)+\operatorname{MLL}\left(S_{j}\right)-\operatorname{MLL}\left(S_{i} \cup S_{j}\right)
$$

where MLL $(\cdot)$ denotes the segment Maximum Log-Likelihood function (MLL). It is the log-likelihood of the segment (pixels in the segment are considered independent realizations) with respect to the assumed PDF (for example, the KummerU distribution) whose parameters are estimated in the ML (hence, the name) sense. Its expression is given by

$$
\operatorname{MLL}(S)=\sum_{i \in S} \ln \left(p_{\mathbf{k}}\left(\mathbf{k}_{i} \mid \theta_{S}\right)\right)
$$

where $\theta_{S}$ represents the set of distribution parameters.

1) GMLL: In general, the covariance matrix and the texture parameters are unknown. One solution consists in replacing the SIRV parameters by their estimates. After replacing the covariance matrix $[M]$ and texture parameters $(m, \mathcal{L}$, and $\mathcal{M}$ for the Fisher PDF) by their respective ML estimators, the SC becomes

$$
\operatorname{SC}_{i, j}=\operatorname{GMLL}\left(S_{i}\right)+\operatorname{GMLL}\left(S_{j}\right)-\operatorname{GMLL}\left(S_{i} \cup S_{j}\right)
$$

where $\operatorname{GMLL}(S)$ is the generalized maximum log-likelihood (GMLL) function for segment $S$. 


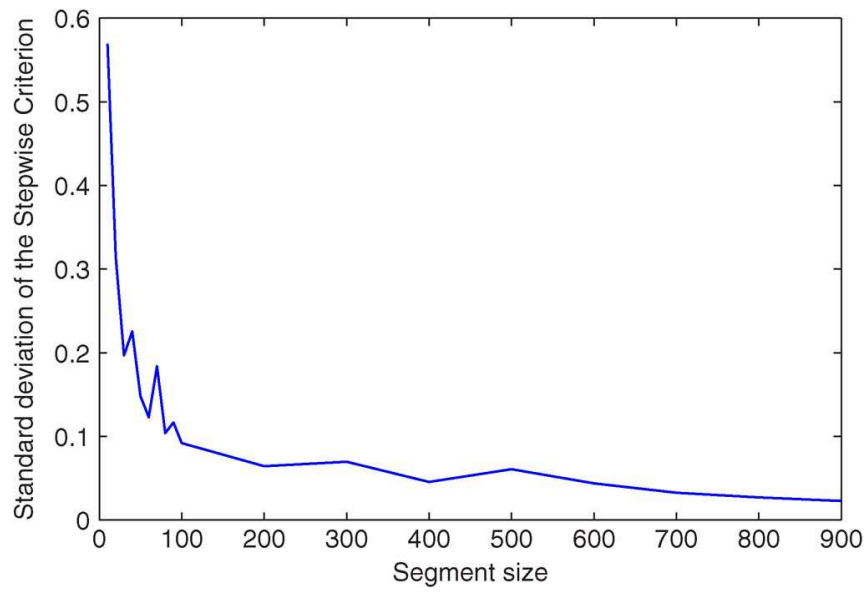

Fig. 4. Standard deviation of the normalized KummerU SC as a function of the window size $N$.

2) For the KummerU PDF: The GMLL function for segment $S$ is derived from (9). It can be rewritten as

$$
\begin{aligned}
& \operatorname{GMLL}(S) \\
&=-p N \ln (\pi)-N \ln \left\{\left|\left[\hat{M}_{M L}\right]\right|\right\} \\
&+N \ln \left\{\frac{\Gamma(\hat{\mathcal{L}}+\hat{\mathcal{M}}) \Gamma(p+\hat{\mathcal{M}})}{\Gamma(\hat{\mathcal{L}}) \Gamma(\hat{\mathcal{M}})}\right\}+p N \ln \left\{\frac{\hat{\mathcal{L}}}{\hat{\mathcal{M}} \hat{m}}\right\} \\
&+\sum_{i \in S} \ln \left\{\mathrm{U}\left(p+\hat{\mathcal{M}} ; 1+p-\hat{\mathcal{L}} ; \frac{\hat{\mathcal{L}}}{\hat{\mathcal{M}} \hat{m}} \mathbf{k}_{i}^{H}\left[\hat{M}_{M L}\right]^{-1} \mathbf{k}_{i}\right)\right\}
\end{aligned}
$$

where $\hat{\mathcal{L}}, \hat{\mathcal{M}}$, and $\hat{m}$ are the ML estimators of the Fisher parameters $\mathcal{L}, \mathcal{M}$, and $m$, respectively. $\left[\hat{M}_{M L}\right]$ is the $\mathrm{ML}$ estimator of $\left[M_{M L}\right]$ for segment $S$ [see (4)].

It can be noticed that the second term of (14) corresponds to the Wishart criterion [12]. All other terms can be viewed as correction terms introduced by the texture modeling of the PolSAR data.

\section{B. Segmentation Results}

To evaluate the potential and limits of the method, the hierarchical segmentation algorithm proposed by Beaulieu and Touzi [12] has been implemented. Fig. 4 shows the standard deviation for the normalized KummerU criterion $(S C / N)$ as a function of the window size $N$. This curve has been plotted for the two regions containing KummerU realizations with two different sets of parameters. As observed in Fig. 4, the standard deviation is stable for a sufficiently large region. For the segments containing less than 50 pixels, the standard deviation increases, probably due to a poor parameter estimation. It yields that a reasonable "minimum window" for the KummerU criterion should contain at least 50 pixels. In the following sections, the Gaussian and KummerU segmentation are tested with both synthetic and high-resolution single-look-complex images.

1) On a Synthetic Image: The synthetic data set consists of an image of $140 \times 140$ pixels. It is composed of six areas. Five of them contain the independent realizations of the
TABLE III

Covariance MATrices of THE SPECKLE (PER AREA)

\begin{tabular}{||c|c|c|c|c|c|c||}
\hline \hline Area & {$[M]_{11}$} & {$[M]_{22}$} & {$[M]_{33}$} & {$[M]_{12}$} & {$[M]_{13}$} & {$[M]_{23}$} \\
\hline \hline 1 & 2.677 & 0.171 & 0.152 & $-0.018+0.064 i$ & $0.131-0.051 i$ & $0.008+0.032 i$ \\
\hline 2 & 2.500 & 0.300 & 0.200 & $-0.018+0.064 i$ & $0.131-0.051 i$ & $0.008+0.032 i$ \\
\hline 3 & 2.677 & 0.171 & 0.152 & $-0.018+0.064 i$ & $0.131-0.051 i$ & $0.008+0.032 i$ \\
\hline 4 & 2.677 & 0.171 & 0.152 & $-0.018+0.064 i$ & $0.131-0.051 i$ & $0.008+0.032 i$ \\
\hline 5 & 2.677 & 0.171 & 0.152 & $-0.018+0.064 i$ & $0.131-0.051 i$ & $0.008+0.032 i$ \\
\hline 6 & 2.677 & 0.171 & 0.152 & $-0.018+0.064 i$ & $0.131-0.051 i$ & $0.008+0.032 i$ \\
\hline \hline
\end{tabular}

TABLE IV

TEXTURE PDF (PER AREA)

\begin{tabular}{||c|c|c|c|c||}
\hline \hline Area & PDF & $\mathcal{L}$ & $\mathcal{M}$ & $\mu$ \\
\hline \hline 1 & dirac & $\infty$ & $\infty$ & 1 \\
\hline 2 & Fisher & 2 & 3 & 1 \\
\hline 3 & Fisher & 2 & 3 & 2 \\
\hline 4 & Fisher & 1 & 3 & 2 \\
\hline 5 & Fisher & 2 & 5 & 2 \\
\hline 6 & Fisher & 2 & 3 & 1 \\
\hline \hline
\end{tabular}

multivariate KummerU distribution [see (9)]. The parameters of the KummerU PDF are different for each of the areas and are given in Tables III and IV. The outer area (class 1) is a special case since its texture is deterministic and constant (equals 1). It follows that the pixels in this area are drawn from a multivariate Gaussian PDF. It can also be viewed as a KummerU PDF with infinite shape parameters $\mathcal{L}$ and $\mathcal{M}$.

Note that the covariance matrices of the speckle satisfy the normalization condition $\operatorname{Tr}([M])=3$. In order to test the segmentation algorithm thoroughly, only a limited set of parameters changes values between two neighboring regions. Thus, the successful segmentations show the ability of the underlying method to distinguish between similar areas.

The Gaussian distribution can be viewed as a particular case of the KummerU distribution (for large shape parameters $\mathcal{L}$ and $\mathcal{M}$ ). It is expected that, asymptotically (for a large number of samples $N$ ), the KummerU segmentation gives at least the same performances as the Gaussian criterion.

The hierarchical segmentation algorithm is initialized with a partition where each segment is a bloc of $10 \times 10$ pixels. The initial partition is composed of 196 segments. The segmentation results based on the Gaussian and KummerU criteria are respectively shown in Fig. 5(c) and (d) with the partitions containing six segments. This example clearly shows that the segmentation based on the KummerU criterion gives the best segmentation results. This is quite logical because the six segments can be viewed as six different KummerU clutter models.

Nevertheless, for purely Gaussian distributed regions, some problems with the KummerU criterion can occur. A synthetic data set composed by three Gaussian distributed regions $\left(S_{1}, S_{2}\right.$, and $\left.S_{3}\right)$ has been generated. $S_{1}$ and $S_{2}$ follow a Gaussian distribution with covariance matrix $\left[M_{1}\right]\left(\mathcal{N}\left(0,\left[M_{1}\right]\right)\right)$, whereas $S_{3}$ has a covariance matrix $\left[M_{2}\right]\left(\mathcal{N}\left(0,\left[M_{2}\right]\right)\right)$. Note that the Gaussian regions are very similar as observed on the Frobenius norm between $\left[M_{1}\right]$ and $\left[M_{2}\right]$ (0.0012). Table V shows the SC for the Gaussian and KummerU criteria between $S_{1}$ and $S_{2}\left(d\left(S_{1}, S_{2}\right)\right)$ and between $S_{1}$ and $S_{3}\left(d\left(S_{1}, S_{3}\right)\right)$. It can be seen that, for a finite number of samples $N$, the KummerU segmentation may fail due to the higher dimensionality of the parameter space. 


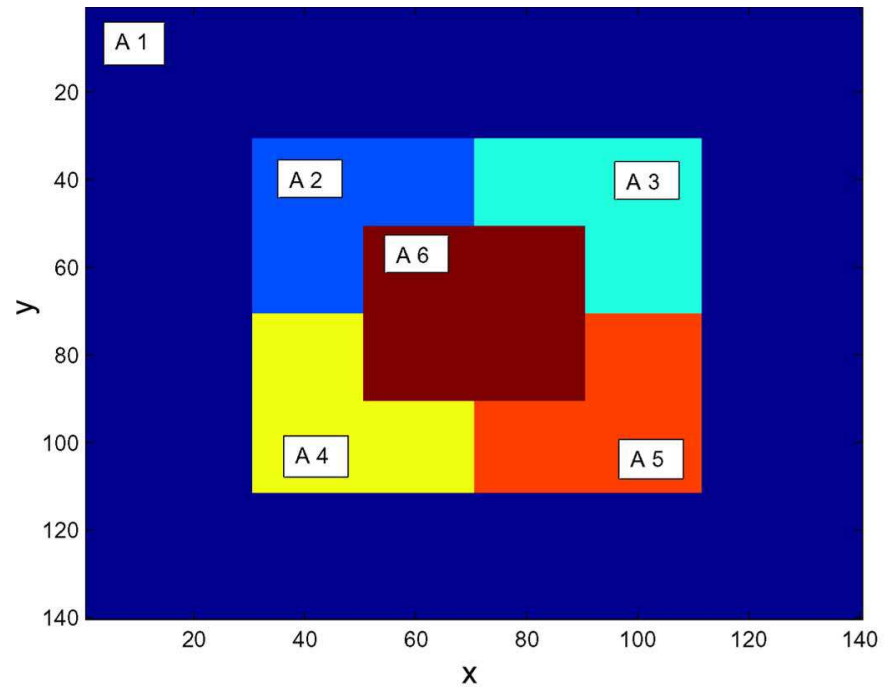

(a)

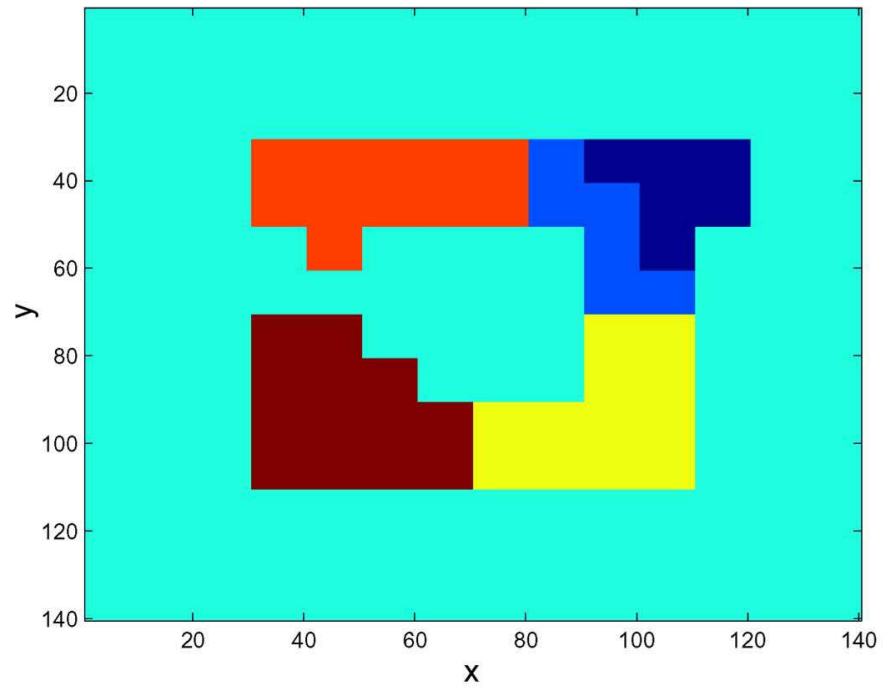

(c)

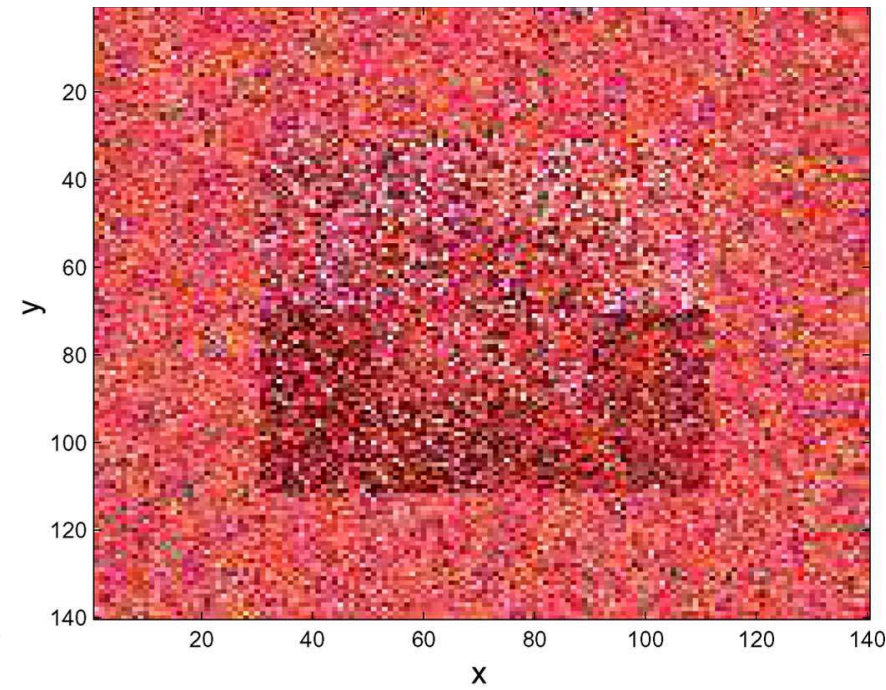

(b)

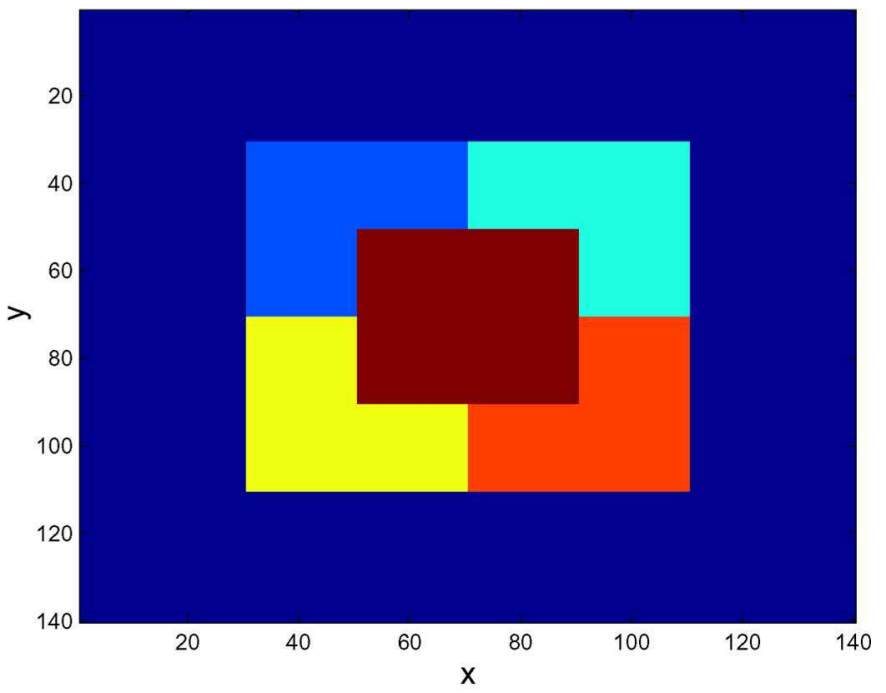

(d)

Fig. 5. Segmentation results of a simulated data set $(140 \times 140$ pixels). (a) Areas of the matrix. (b) Colored composition of the target vector in the Pauli basis $[k]_{1}-[k]_{3}-[k]_{2}$. Partitions containing six segments: (c) Gaussian criterion and (d) KummerU criterion.

TABLE V

SC FOR GAUSSIAN REGIONS

\begin{tabular}{||c|c|c||}
\hline \hline SC & $d\left(S_{1}, S_{2}\right)$ & $d\left(S_{1}, S_{3}\right)$ \\
\hline Gaussian & 2.443 & 2.975 \\
\hline KummerU & 5.322 & 2.702 \\
\hline \hline
\end{tabular}

2) On High-Resolution L-Band Data: In this part, a forested area $(500 \times 400$ pixels $)$ over the Oberpfaffenhofen test site (ESAR, L-band) has been segmented. The initial partition is composed of 2000 segments where each segment is a bloc of $10 \times 10$ pixels. The segmentation results with the Gaussian and KummerU criteria are shown in Fig. 6(a) and (b), respectively.

For the Gaussian criterion, only the determinant of the sample covariance matrix is taken into account. Both the structure of the covariance matrix and the power (texture) of the clutter are ignored. Consequently, the Gaussian criterion cannot distinguish between two regions having different texture values and/or covariance matrices whose determinants are equal but which have different structures. The KummerU criterion uses information about the texture and full information about the

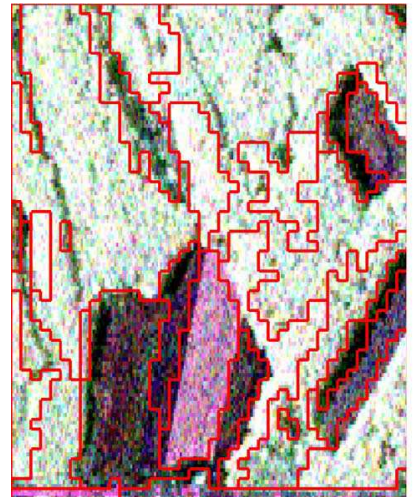

(a)

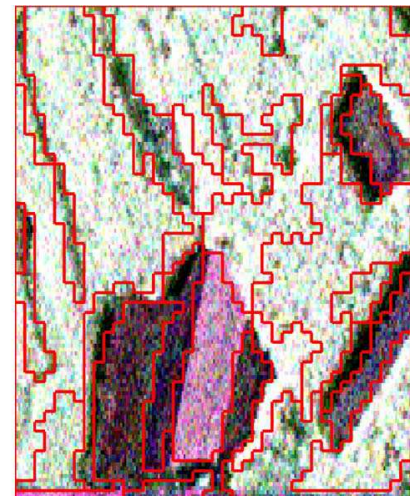

(b)
Fig. 6. Segmentation results for the L-band ESAR data over the Oberpfaffenhofen test site $(500 \times 400$ pixels). Partitions containing 30 segments over a colored composition of the target vector in the Pauli basis $[k]_{1}-[k]_{3}-[k]_{2}$ : (a) Gaussian criterion and (b) KummerU criterion.

covariance matrix and is able to give a better segmentation of heterogeneous scenes, such as forested areas, as observed in Fig. 6(b). 


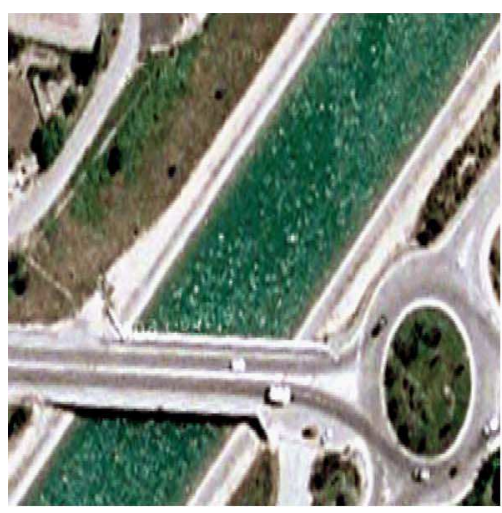

(a)

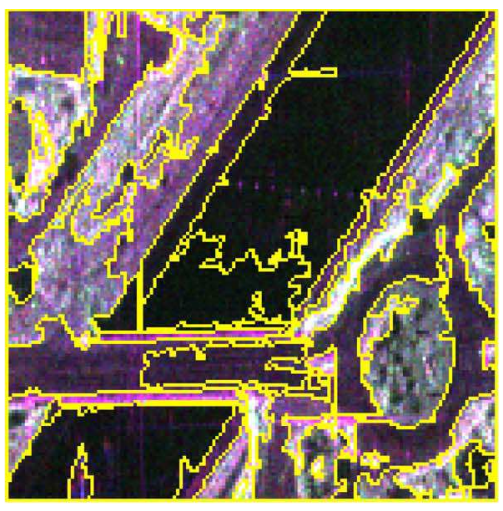

(b)

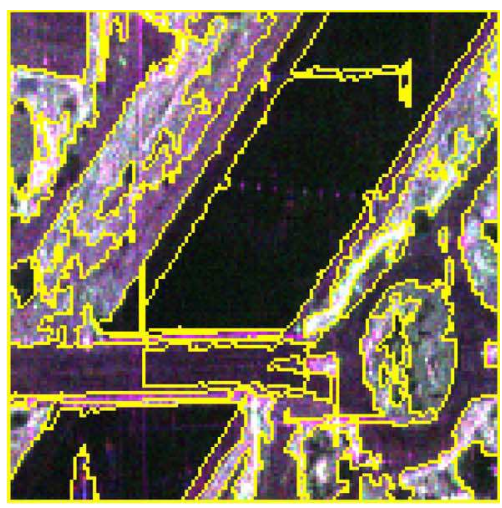

(c)

Fig. 7. Segmentation results for the X-band RAMSES data over the Salon de Provence test site $(1050 \times 1050$ pixels $)$. Partitions containing 55 segments over a colored composition of the target vector in the Pauli basis $[k]_{1}-[k]_{3}-[k]_{2}$ : (a) Optical image Centre National d'Etudes Spatiales/spot image, (b) Gaussian criterion, and (c) KummerU criterion.

3) On Very High Resolution X-Band Data: In this section, a very high resolution data set acquired by the X-band RAMSES sensor over the Salon de Provence test site with a resolution of $10 \mathrm{~cm}$ is analyzed. Fig. 7(a) shows an optical image of the test site. The segmentation results with the Gaussian and KummerU criteria are shown in Fig. 7(b) and (c), respectively. The segmentation algorithm is initialized with a partition where each segment is a bloc of $7 \times 7$ pixels.

From this data set, it can be noticed that more features are segmented in the traffic circle with the KummerU criterion than with the Gaussian criterion. Moreover, the artifact in the water (on the northeast of the image) is better retrieved with the KummerU segmentation. Concerning the water itself, the Gaussian criterion leads to an oversegmented partition, particularly near the bridge.

\section{Determining the Number of Clusters Automatically}

\section{A. General Context}

Based on the initial partition, the hierarchical segmentation iteratively merges the segments that are both statistically similar (MLL criterion) and spatially close (neighboring condition). The procedure may continue until a partition with a single segment, comprising the whole image, is obtained.

Note that the hierarchical segmentation algorithm is generally suboptimal since neither the initial partition nor the iterative merging of the segments is guaranteed to be optimal in the general sense (i.e., with respect to maximizing the sum of the segments' log-likelihood), although the iterative merging is still the optimal processing for the imposed initial partitioning and a segment neighboring condition.

Summarily, it is useful to describe the hierarchical segmentation method as an iterative transition from the oversegmented partitioning to an undersegmented one. Each iteration results in an intermediate partition, and thus, a sequence of partitions is created. It would be valuable to determine the most appropriate partition in this sequence, with respect to some optimality criterion.

It is, however, difficult to decide between over- and undersegmentation, except for the carefully chosen images [25],
[26]. As stated in [27], the ideal segmentation maximizes the homogeneity of the retained data clusters while ensuring that they are as dissimilar as possible. Such a perfect tradeoff is, however, difficult to obtain since, during the region merging (as what happens in the hierarchical segmentation algorithms), both the homogeneity of the clusters and the dissimilarity between them become weaker.

This problem is encountered in most of the data clustering and image segmentation algorithms, and a number of techniques have been applied (a comprehensive review is found in [28]). However, some of the techniques are not applicable for many SAR images. For example, the solution retained in [27] is to incorporate the boundary curvature ratio, region homogeneity, and boundary smoothness into a single merging criterion, which is also used to estimate and to threshold the intervariance of the obtained data clusters. This is not appropriate for the SAR images for two reasons: First, many regions of interest in the SAR images have irregular shapes (forests, lakes, buildings, etc.), and second, the textured characteristic of the SAR images makes both the boundary smoothness and the region homogeneity unreliable information. The latter remark is in line with preferring stochastic approaches over contour approaches in the SAR image segmentation.

\section{B. Between-Cluster Entropy}

The use of the information theory framework, closely related to the retained statistical description of the SAR images, has been attempted first. The cluster evaluation function, defined in [29], is used in [30] as a measure for the between-cluster entropy (intervariance). Formally, for all pairs of pixels (vectors) $\mathbf{x}_{\mathbf{i}}$ and $\mathbf{x}_{\mathbf{j}}$ in a partitioned image, a membership function $M\left(\mathbf{x}_{\mathbf{i}}, \mathbf{x}_{\mathbf{j}}\right)$ is defined, which equals one if $\mathbf{x}_{\mathbf{i}}$ and $\mathbf{x}_{\mathbf{j}}$ belong to different clusters (referred as segments under the terminology used in this paper) and zero if they belong to the same cluster. Thus, the between-cluster entropy is defined as

$$
\begin{aligned}
H & =-\log (V) \\
V & =\frac{1}{2 \prod_{k=1}^{K} N_{k}} \sum_{i=1}^{N} \sum_{j=1}^{N} M\left(\mathbf{x}_{\mathbf{i}}, \mathbf{x}_{\mathbf{j}}\right) G\left(\mathbf{x}_{\mathbf{i}}-\mathbf{x}_{\mathbf{j}}, 2 \sigma^{2} \mathbf{I}\right) .
\end{aligned}
$$




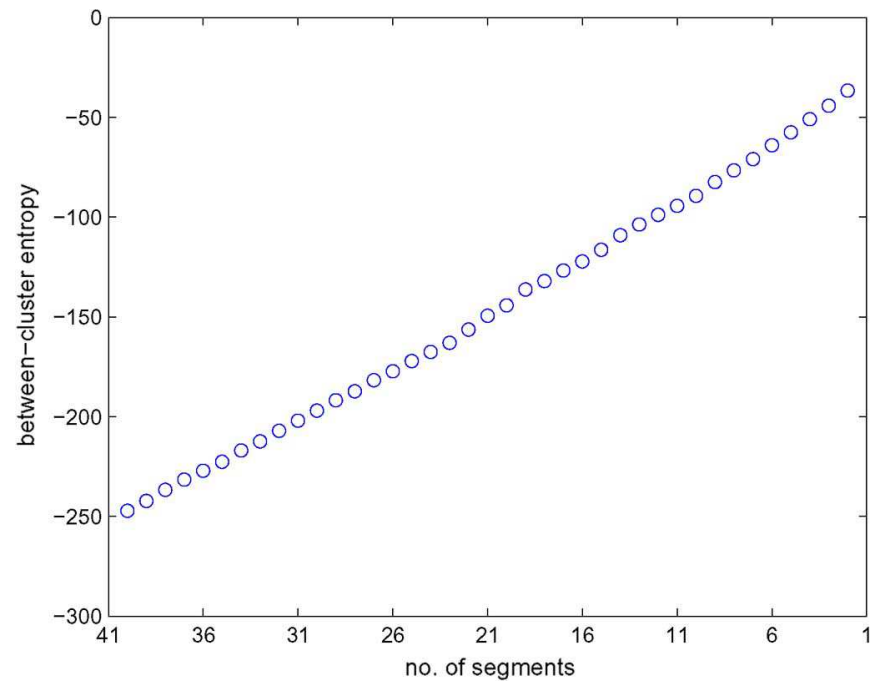

Fig. 8. Between-cluster entropy for the iterative hierarchical KummerU segmentation.

In the aforementioned equations, $\sigma$ is a positive parameter, namely, the width of the centered Gaussian kernel $G(\cdot)$ used in computing the nonparametric Parzen estimate of the PDF and, consequently, the estimate of Renyi's quadratic (obtained for $\alpha=2$ ) entropy. The between-cluster entropy is defined, by analogy, on the basis of this estimate.

Note that the between-cluster entropy equals $+\infty$ for the ending one-segment partition (indeed, the membership function $M\left(\mathbf{x}_{\mathbf{i}}, \mathbf{x}_{\mathbf{j}}\right)$ is always zero in that case and so is $\left.V\right)$. On the other hand, if many segments tend to be very close such that the (norm of the) difference $\mathbf{x}_{\mathbf{i}}-\mathbf{x}_{\mathbf{j}}$ is rather small (consequently, the value of $G\left(\mathbf{x}_{\mathbf{i}}-\mathbf{x}_{\mathbf{j}}, 2 \sigma^{2} \mathbf{I}\right)$ is large) while $M\left(\mathbf{x}_{\mathbf{i}}, \mathbf{x}_{\mathbf{j}}\right)$ equals one, the value of the between-cluster entropy is small. The latter occurs in oversegmented images, for example, in the initial partition of the considered hierarchical segmentation method. Consequently, it is expected for the between-cluster entropy to start with small values and, as the segments are hierarchically merged, to progressively increase.

Jenssen et al. use the variation of the between-cluster entropy quantity (during an iterative clustering process) to automatically find the true number of clusters [30]. Specifically, a sudden increase is observed in the between-cluster entropy when the number of clusters is reduced to a value less than the true number of the clusters (i.e., the partitioning goes into undersegmentation). A similar behavior was expected for the PolSAR hierarchical segmentation problem.

To this end, the between-cluster entropy has been computed for each partitioning issued during the iterative hierarchical segmentation proposed in this paper. A value of 0.1 has been considered for $\sigma$. The obtained values for the hierarchical segmentation of the considered synthetic PolSAR image [see Fig. 5(a)] are presented in Fig. 8.

While the between-cluster entropy is constantly increasing, as expected, unfortunately, no sudden change seems to occur in the vicinity of the desired number of segments (namely, six).

Several justifications may be advanced for this behavior. First, unlike the data clusters considered in [30], which are well separated geometrically (belonging to disjoint geometrical regions), the data in the six areas of the considered synthetic
PolSAR image are not. Specifically, while the vectors are drawn from different PDFs for each area, their ranges superpose.

\section{L-Method}

Another approach has been then considered, namely, the use of the L-method [28]. This method uses the very error (quality) function that is used to perform the cluster merging during the hierarchical segmentation algorithm, specifically the LogLikelihood Function (LLF) of the partition (i.e., the sum of the MLL values for all the segments of the partition). As this is readily computed during the proposed method, no further computational effort is required. The knee of this error function is identified, and the optimal number of clusters is chosen at that point. The knee of a curve is somewhat similar to the point of the maximum curvature.

The interest for the particular knee point comes from the fact that it separates the two linearlike parts of the error function graph. For example, the proposed hierarchical segmentation algorithm starts by merging the relatively similar clusters and the trend; thus, the error (quality) function slowly increases. This remains almost unchanged during the oversegmentation. Anyway, after the correct number of segments is reached and the undersegmentation begins, the dissimilar segments begin to be merged. As such, the homogeneity of the newly obtained segments drops fast, and the quality function of the partition degrades rapidly. Obviously, the optimal tradeoff lies somewhere between the over- and undersegmented partitions.

The L-method divides the graph of the error (quality) function into two parts and approximates each part with a straight line. The pair of lines that most closely fit the error function curve is retained, and their junction point is the looked-for knee. The best pair of lines is that which minimizes the weighted sum of approximation errors for the two parts of the error function graph.

For many images, the hierarchical segmentation algorithms spend many iterations in the oversegmentation since it usually starts from an overrefined, sometimes even pixel level, partition of the image. As this tends to unbalance the error contributions of the over- and undersegmented parts in determining the knee of the error curve, it is reasonable to do both of the following: 1) Drop some of the initial iterations of the hierarchical segmentation algorithms, and 2) use an iterative procedure in searching for the knee. The first step relocates the searching of the knee toward the final steps of the algorithm (toward the undersegmented region) while the second step refines the found knee by considering a converging procedure toward a stable procedure. Specifically, after a knee is found, the search is performed again, the focus region being adjusted so that the previously found knee is in the middle. The procedure is described in [28].

1) On Synthetic Image: First, we present the results obtained with the L-method for the synthetic six-area image. The quality functions during the hierarchical segmentation algorithm are shown in Fig. 9.

While the number of the optimal number of segments [see (6)] for the considered image is not accurately retrieved by the L-method, the estimate is quite close [see (5)]. This 


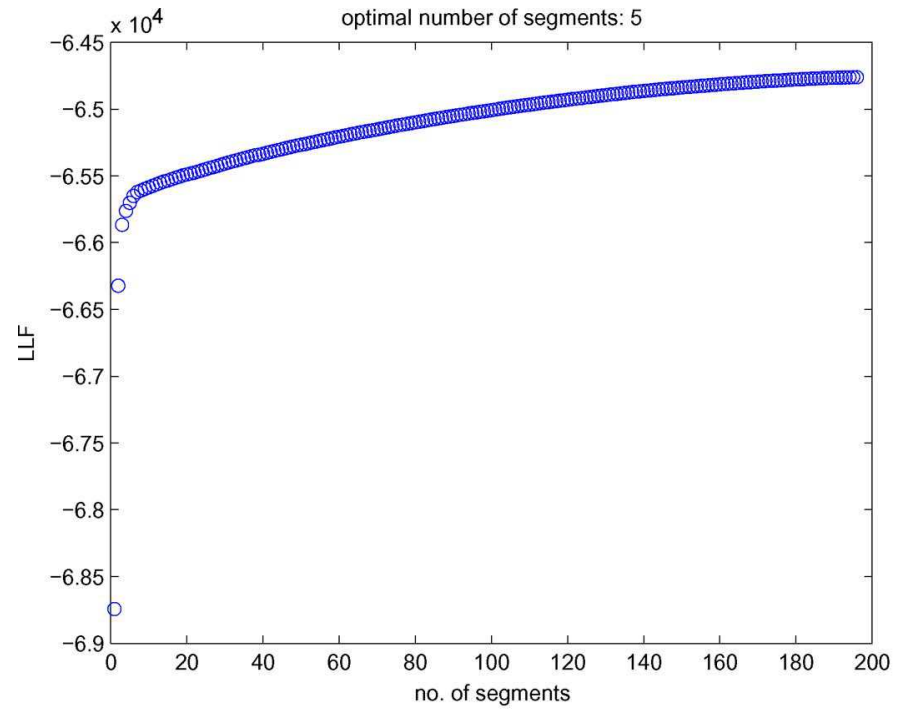

(a)
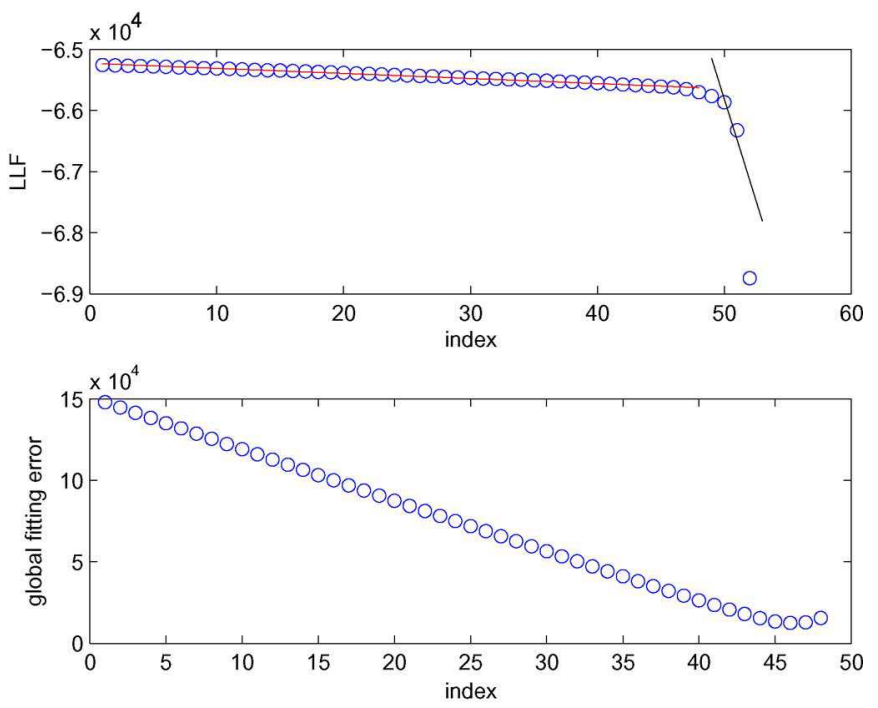

(b)

Fig. 9. Determination of the final partition for the KummerU segmentation on the synthetic image. (a) LLF. (b) LLF and global fitting error for the last 50 iterations.

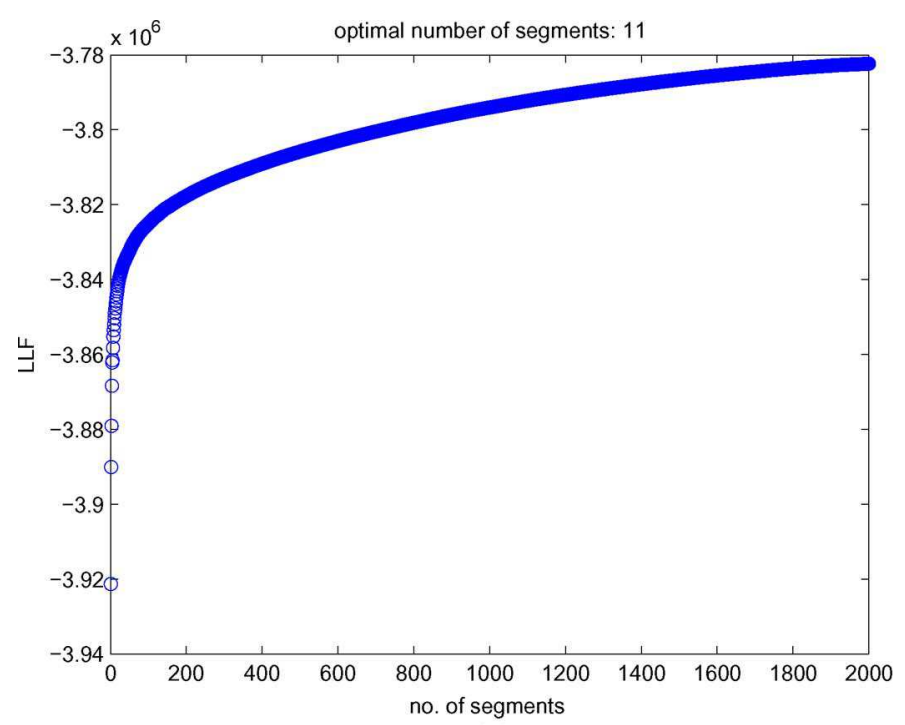

(a)
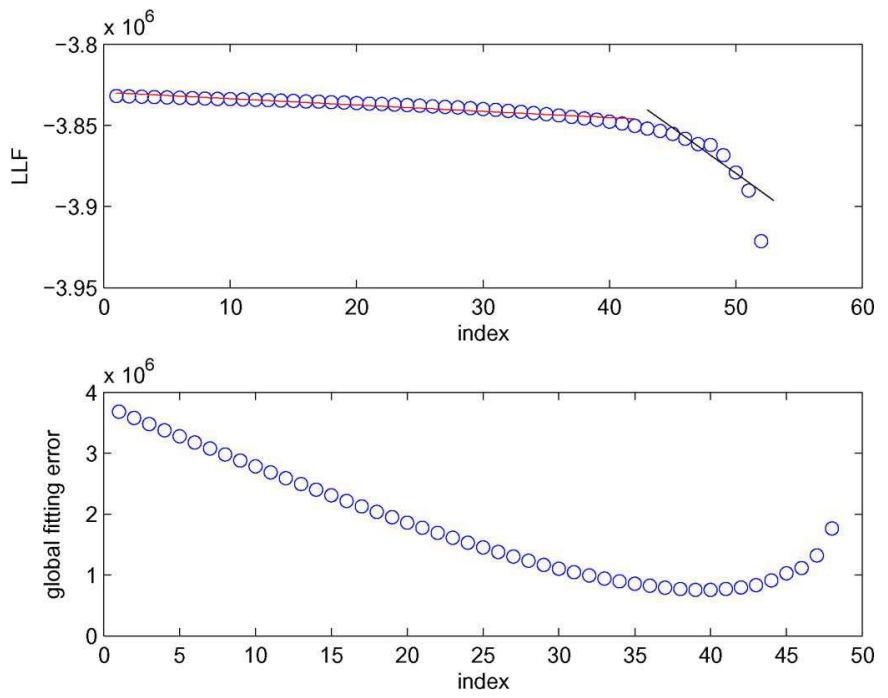

(b)

Fig. 10. Determination of the final partition for the KummerU segmentation over the Oberpfaffenhofen test site. (a) LLF. (b) LLF and global fitting error for the last 50 iterations.

number has been determined using the noniterative version of the L-method, applied to a focus region containing the last 50 iterations of the hierarchical segmentation algorithm [meaning, the leftmost 50 points of the graphs in Fig. 9(a)].

The application of the L-method under these conditions is illustrated in Fig. 9(b). The upper graphs show the dual-line approximation of the quality function in the optimal position while the lower graphs present the variation of the global (weighted) fitting error.

2) On High-Resolution L-Band Data: Using the L-method for automatically determining the optimal number of segments for the real images has been also assessed. The results are presented for the Oberpfaffenhofen image, as shown in Fig. 10. Note that the identified optimal number of segments is around ten. The noniterative L-method has been applied to a focus region containing the last 50 iterations of the hierarchical segmentation algorithm [meaning, the leftmost 50 points of the graphs in Fig. 10(a)]. The application of the L-method under these conditions is illustrated in Fig. 10(b).

Finally, the iterative version of the L-method has been applied on the same data set (the Oberpfaffenhofen image) in order to test the convergence properties of this procedure. We found a relatively fast convergence. Indeed, for the KummerU segmentation, the optimal number of segments was found to be through the series $[508 \rightarrow 191 \rightarrow 65 \rightarrow 22 \rightarrow 9$ ]. The final value is quite close to that obtained in the previous case, when the noniterative L-method has been applied.

Note, however, that the optimal segmentation is still a very subjective notion and that the L-method is not completely objective since the notion of the knee is quite loose and, more, is not necessarily the most appropriate from the user point of view (the same concerns the hierarchical segmentation algorithm). 


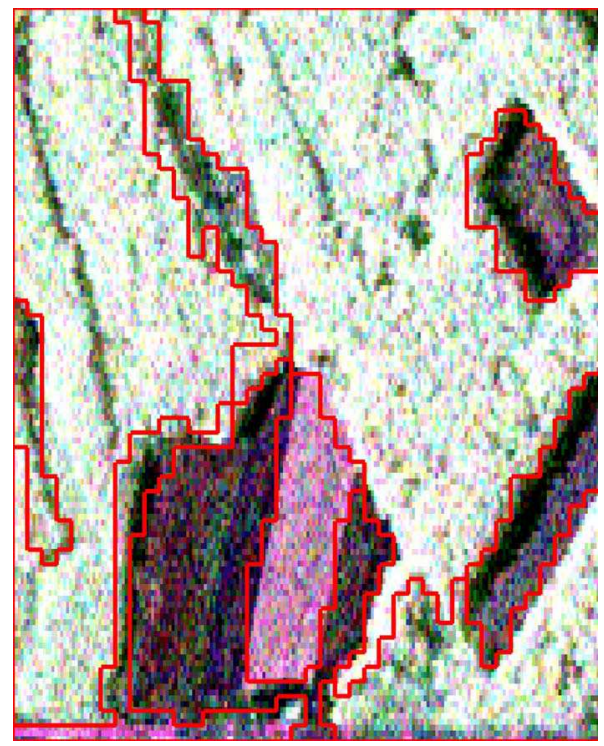

Fig. 11. Segmentation results with the KummerU criterion for the L-band ESAR data over the Oberpfaffenhofen test site $(500 \times 400$ pixels $)$. Partition containing ten segments over a colored composition of the target vector $[k]_{1}-$ $[k]_{3}-[k]_{2}$

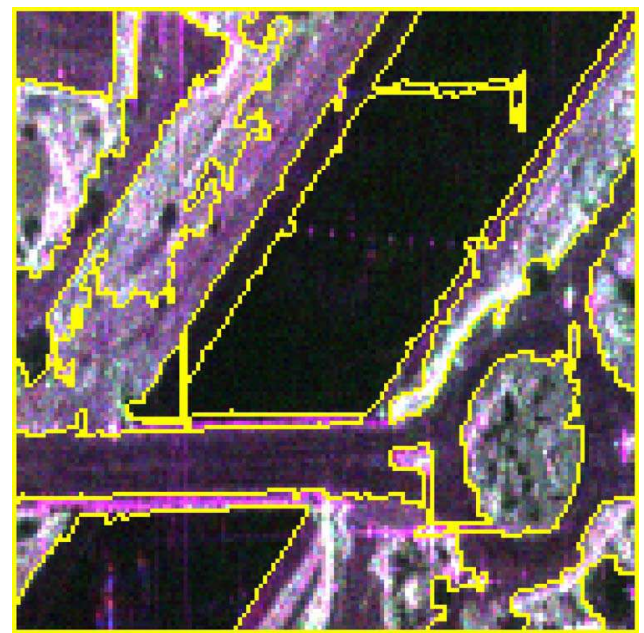

Fig. 12. Segmentation results with the KummerU criterion for the X-band RAMSES data over the Salon de Provence test site $(1050 \times 1050$ pixels). Partition containing 19 segments over a colored composition of the target vector $[k]_{1}-[k]_{3}-[k]_{2}$.

For illustrative purposes, the partition of the Oberpfaffenhofen image with ten segments (in the range of those obtained using the L-method) is presented in Fig. 11.

3) On Very High Resolution X-Band Data: In this part, the iterative version of the L-method has been applied on the $\mathrm{X}$-band RAMSES data set over the Salon de Provence test site. For the KummerU segmentation, the optimal number of segments was found to be through the series $[7298 \rightarrow 3889 \rightarrow$ $1472 \rightarrow 457 \rightarrow 147 \rightarrow 52 \rightarrow 21]$. The partition containing the optimal estimated number of segments is shown in Fig. 12.

It has been shown that the L-method provides an objective and pertinent criterion for automatically identifying the optimal number of segments in the hierarchical segmentation procedure. A more in-depth analysis is given in [28].

\section{CONCLUSION}

In this paper, the authors have proposed to apply the SIRV estimation scheme to derive the covariance matrix and the texture parameter. By rewriting the texture variable as the product of a mean backscattered power $\mu$ with a normalized texture component $\xi$, the Beta prime PDF has been introduced to characterize the $\xi$ variable. In this case, the texture parameter $\tau$ is Fisher distributed, and the target scattering vector follows a KummerU PDF. The asymptotic cases of this multivariate distribution have been studied. It generalizes the well-known $\mathcal{K}$ and $\mathcal{G}^{0}$ distributions. Some experiments at the X-, C-, L-, and $\mathrm{P}$-bands have shown that this new statistical model is well adapted to fit a wide range of PolSAR clutters.

Based on those considerations, the multivariate KummerU distribution has been implemented in an ML hierarchical segmentation algorithm. The segmentation results on the synthetic and real PolSAR data have shown that the SIRV estimation scheme combined with the KummerU PDF provides the best performances compared to the classical Gaussian criterion. Next, a method based on the knee of the LLF has been implemented to determine automatically the "optimal" number of segments in the final partition.

Further works will deal with the use of texture and polarimetric informations for the ML texture tracking with highresolution PolSAR data.

\section{APPENDIX A \\ CONVERGENCE OF THE KUMMERU PDF TOWARD THE $\mathcal{K}$ PDF}

For large $\mathcal{M}$, the Fisher PDF tends toward the Gamma PDF. This appendix shows a proof of the convergence of the KummerU PDF toward the $\mathcal{K}$ PDF as $\mathcal{M}$ tends toward infinity. Abramowitz and Stegun have shown the following relation [see 31, Eq. 13.3.3] which links an asymptotic case of the KummerU function with the modified Bessel function of the second kind (denoted by BesselK).

$$
\lim _{a \rightarrow \infty}\{\Gamma(1+a-b) U(a, b, z / a)\}=2 z^{\frac{1}{2}-\frac{b}{2}} \operatorname{BesselK}_{b-1}(2 \sqrt{z})
$$

Let $\quad a=p+\mathcal{M}, \quad b=1+p-\mathcal{L}, \quad$ and $\quad z=(p+$ $\mathcal{M} / \mathcal{M} m) \mathcal{L} \mathbf{k}^{H}[M]^{-1} \mathbf{k}$. By injecting the expression of $a$, $b$, and $z$ in (16) and taking the limit when $\mathcal{M}$ tends toward infinity, it yields

$$
\begin{aligned}
\lim _{\mathcal{M} \rightarrow \infty} & \left\{\Gamma(\mathcal{L}+\mathcal{M}) U\left(p+\mathcal{M}, 1+p-\mathcal{L}, \frac{\mathbf{k}^{H}[M]^{-1} \mathbf{k} \mathcal{L}}{\mathcal{M} m}\right)\right\} \\
= & 2\left[\frac{\mathcal{L} \mathbf{k}^{H}[M]^{-1} \mathbf{k}}{m}\right]^{\frac{1}{2}-\frac{(1+p-\mathcal{L})}{2}} \\
& \times \operatorname{BesselK}_{p-\mathcal{L}}\left(2 \sqrt{\frac{L \mathbf{k}^{H}[M]^{-1} \mathbf{k}}{m}}\right) .
\end{aligned}
$$

Moreover, one can easily prove that

$$
\lim _{\mathcal{M} \rightarrow \infty} \frac{\Gamma(p+\mathcal{M})}{\Gamma(\mathcal{M}) \mathcal{M}^{p}}=1 .
$$


By combining (17) and (18) in (9), we retrieve the expression of the $\mathcal{K}$ distribution

$$
\begin{aligned}
p_{\mathbf{k}}(\mathbf{k} \mid[M], \mathcal{L}, m)= & \frac{2}{\pi^{p} \Gamma(\mathcal{L})|[M]|}\left(\frac{\mathcal{L}}{m}\right)^{\left(\frac{\mathcal{L}+p}{2}\right)} \\
& \times\left[\mathbf{k}^{H}[M]^{-1} \mathbf{k}\right]^{\left(\frac{\mathcal{L}-p}{2}\right)} \\
& \times \text { BesselK }_{p-\mathcal{L}}\left(2 \sqrt{\frac{\mathcal{L} \mathbf{k}^{H}[M]^{-1} \mathbf{k}}{m}}\right) .
\end{aligned}
$$

\section{APPENDiX B \\ ML FISHER PARAMETER ESTIMATION}

The scalar texture parameter $\tau$ is the random power of the clutter; it characterizes the randomness induced by the variations in the radar backscattering over different realizations (pixels). This scalar texture parameter is assumed to be independent from the polarization channel. For pixel $i$, it can be rewritten as the product of a normalized texture parameter $\xi_{i}$ with the mean backscattered power $\mu$ by

$$
\tau_{i}=\mu \xi_{i}
$$

where $\mu$ is assumed as a deterministic quantity (parameter). Note that the current sample $i$ is excluded from the computation of $\mu$. Its expression is given by

$$
\mu=\frac{1}{N-1} \sum_{\substack{j=1 \\ j \neq i}}^{N} \tau_{j}
$$

By inserting (2) and (21) in (20), one can express $\xi_{i}$ as the following ratio:

$$
\xi_{i}=\frac{\tau_{i}}{\mu}=\frac{\tau_{i}}{\frac{1}{N-1} \sum_{\substack{j=1 \\ j \neq i}}^{N} \tau_{j}}=\frac{\mathbf{k}_{i}^{H}[M]^{-1} \mathbf{k}_{i}}{\frac{1}{N-1} \sum_{\substack{j=1 \\ j \neq i}}^{N} \mathbf{k}_{j}^{H}[M]^{-1} \mathbf{k}_{j}} .
$$

If $\tau$ follows a Fisher PDF, denoted by $\mathcal{F}[\cdot]$, one can prove that

$$
\mathcal{F}\left[\tau \mid m=\frac{\mu \mathcal{L}}{\mathcal{M}}, \mathcal{L}, \mathcal{M}\right]=\mu \mathcal{B P}[\xi \mid \mathcal{L}, \mathcal{M}] .
$$

It yields that $\xi$ follows a Beta prime PDF, denoted by $\mathcal{B P}[\cdot]$. The ML estimators of the Beta prime PDF parameters do not have an analytical expression. They are the solution of the following two equations, which contain the digamma function $\Psi(\cdot)$ by

$$
\begin{aligned}
\Psi(\hat{\mathcal{L}})-\Psi(\hat{\mathcal{L}}+\hat{\mathcal{M}}) & =\frac{1}{N} \sum_{i=1}^{N} \ln \left(\frac{\xi_{i}}{1+\xi_{i}}\right) \\
\Psi(\hat{\mathcal{M}})-\Psi(\hat{\mathcal{L}}+\hat{\mathcal{M}}) & =\frac{1}{N} \sum_{i=1}^{N} \ln \left(\frac{1}{1+\xi_{i}}\right) .
\end{aligned}
$$

Next, the transformation $A=\xi /(1+\xi)$ is applied on the data. Indeed, if $\xi \sim \mathcal{B P}(\mathcal{L}, \mathcal{M})$, then $A \sim \mathcal{B}(\mathcal{L}, \mathcal{M})$ where $\mathcal{B}(\cdot)$ is the Beta PDF. The Beta shape parameters $(\mathcal{L}$ and $\mathcal{M})$ are then numerically estimated by solving the ML equations [32]. Finally, the scale parameter $m$ is estimated by the relation $m=\mu \mathcal{L} / \mathcal{M}$.

\section{ACKNOWLEDGMENT}

The authors would like to thank Prof. J. M. Beaulieu (Laval University, Canada) for the very fruitful discussions and advices. The authors would also like to thank the German Aerospace Center (DLR) and the French Aerospace Lab (ONERA) for providing the high and very high resolution PolSAR images. The authors would also like to thank the anonymous reviewers for their helpful comments and suggestions.

\section{REFERENCES}

[1] Y. Delignon, R. Garello, and A. Hillion, "Statistical modelling of ocean SAR images," Proc. Inst. Elect. Eng.-Radar, Sonar Navig., vol. 144, no. 6, pp. 348-354, Dec. 1997.

[2] C. Tison, J.-M. Nicolas, F. Tupin, and H. Maître, "A new statistical model for Markovian classification of urban areas in high-resolution SAR images," IEEE Trans. Geosci. Remote Sens., vol. 42, no. 10, pp. 2046-2057, Oct. 2004.

[3] F. Galland, J.-M. Nicolas, H. Sportouche, M. Roche, F. Tupin, and P. Réfrégier, "Unsupervised synthetic aperture radar image segmentation using Fisher distributions," IEEE Trans. Geosci. Remote Sens., vol. 47, no. 8, pp. 2966-2972, Aug. 2009.

[4] S. Cloude and E. Pottier, "An entropy based classification scheme for land applications of polarimetric SAR," IEEE Trans. Geosci. Remote Sens., vol. 35, no. 1, pp. 68-78, Jan. 1997.

[5] A. Freeman and S. Durden, "A three-component scattering model for polarimetric SAR data," IEEE Trans. Geosci. Remote Sens., vol. 36, no. 3, pp. 963-973, May 1998.

[6] R. Touzi, "Target scattering decomposition in terms of roll-invariant target parameters," IEEE Trans. Geosci. Remote Sens., vol. 45, no. 1, pp. 73-84, Jan. 2007.

[7] J. Lee, M. Grunes, T. Ainsworth, L. Du, D. Schuler, and S. Cloude, "Unsupervised classification using polarimetric decomposition and the complex Wishart classifier," IEEE Trans. Geosci. Remote Sens., vol. 37, no. 5, pp. 2249-2258, Sep. 1999.

[8] H. Skriver, J. Schou, A. Nielsen, and K. Conradsen, "Polarimetric segmentation using the comple Wishart test statistic," in Proc. IGARSS, Toronto, ON, Canada, 2002, vol. 2, pp. 1011-1013.

[9] S. Anfinsen, T. Eltoft, and A. Doulgeris, "A relaxed Wishart model for polarimetric SAR data," in Proc. PolInSAR, Frascati, Italy, 2009.

[10] S. Quegan and I. Rhodes, "Statistical models for polarimetric SAR data," in Proc. IEE Semin. Texture Anal. Radar Sonar, 1993, pp. 8/1-8/8.

[11] J. Lee, D. Schuler, R. Lang, and K. Ranson, "K-distribution for multi-look processed polarimetric SAR imagery," in Proc. IGARSS, Pasadena, CA, 1994, pp. 2179-2181.

[12] J.-M. Beaulieu and R. Touzi, "Segmentation of textured polarimetric SAR scenes by likelihood approximation," IEEE Trans. Geosci. Remote Sens., vol. 42, no. 10, pp. 2063-2072, Oct. 2004.

[13] C. Freitas, A. Frery, and A. Correia, "The polarimetric G distribution for SAR data analysis," Environmetrics, vol. 16, no. 1, pp. 13-31, 2005.

[14] L. Bombrun and J.-M. Beaulieu, "Fisher distribution for texture modeling of polarimetric SAR data," IEEE Geosci. Remote Sens. Lett., vol. 5, no. 3, pp. 512-516, Jul. 2008.

[15] L. Bombrun, J.-M. Beaulieu, G. Vasile, J.-P. Ovarlez, F. Pascal, and M. Gay, "Hierarchical segmentation of polarimetric SAR images using heterogeneous clutter models," in Proc. IGARSS, Cape Town, South Africa, 2009, vol. 3, pp. 5-8.

[16] K. Yao, "A representation theorem and its applications to sphericallyinvariant random processes," IEEE Trans. Inf. Theory, vol. IT-19, no. 5, pp. 600-608, Sep. 1973.

[17] G. Vasile, J.-P. Ovarlez, F. Pascal, and C. Tison, "Coherency matrix estimation of heterogeneous clutter in high resolution polarimetric SAR images," IEEE Trans. Geosci. Remote Sens., vol. 48, no. 4, pp. 18091826, Apr. 2010.

[18] F. Pascal, Y. Chitour, J. P. Ovarlez, P. Forster, and P. Larzabal, "Covariance structure maximum-likelihood estimates in compound Gaussian noise: Existence and algorithm analysis," IEEE Trans. Signal Process., vol. 56, no. 1, pp. 34-48, Jan. 2008.

[19] F. Pascal, P. Forster, J. P. Ovarlez, and P. Larzabal, "Performance analysis of covariance matrix estimates in impulsive noise," IEEE Trans. Signal Process., vol. 56, no. 6, pp. 2206-2217, Jun. 2008.

[20] K. Fang, S. Kotz, and K. Ng, Symmetric Multivariate and Related Distributions. London, U.K.: Chapman \& Hall, 1990. 
[21] S. Zozor and C. Vignat, "Some results on the denoising problem in the elliptically distributed context," IEEE Trans. Signal Process., vol. 58, no. 1, pp. 134-150, Jan. 2010.

[22] Y. Chitour and F. Pascal, "Exact maximum likelihood estimates for SIRV covariance matrix: Existence and algorithm analysis," IEEE Trans. Signal Process., vol. 56, no. 10, pp. 4563-4573, Oct. 2008.

[23] J.-M. Nicolas, "Introduction aux Statistiques de deuxième espèce: Applications des Logs-moments et des Logs-cumulants à l'analyse des lois d'images radar," Traitement du Signal, vol. 19, no. 3, pp. 139-167, 2002.

[24] J.-M. Nicolas, "Application de la transformée de Mellin: Étude des lois statistiques de l'imagerie cohérente," TELECOM ParisTech, Paris, France, Rapport de Recherche, 2006D010, 2006.

[25] J. Lee, M. Grunes, E. Pottier, and L. Ferro-Famil, "Segmentation of polarimetric SAR images," in Proc. IGARSS, Sydney, Australia, 2001, pp. 414-416.

[26] J. Morio, F. Goudail, X. Dupuis, P. Dubois-Fernandez, and P. Réfrégier, "Polarimetric and interferometric SAR image partition into statistically homogeneous regions based on the minimization of the stochastic complexity," IEEE Trans. Geosci. Remote Sens., vol. 45, no. 11, pp. 35993609, Nov. 2007.

[27] X. He, N. H. C. Yung, K. P. Chow, F. Y. L. Chin, R. H. Y. Chung, K.-Y. K. Wong, and K. S. H. Tsang, "Watershed segmentation with boundary curvature ratio based merging criterion," in Proc. 9th IASTED Int. Conf. SIP, Honolulu, HI, 2007, pp. 7-12.

[28] S. Salvador and P. Chan, "Determining the number of clusters/segments in hierarchical clustering/segmentation algorithms," in Proc. 16th IEEE ICTAI, 2004, pp. 576-584.

[29] E. Gokcay and J. Principe, "Information theoretic clustering," IEEE Trans. Pattern Anal. Mach. Intell., vol. 24, no. 2, pp. 158-171, Feb. 2002.

[30] R. Jenssen, K. Hild, J. Principe, and T. Eltoft, "Clustering using Renyi's entropy," in Proc. Int. Joint Conf. Neural Netw., 2003, vol. 1, pp. 523-528.

[31] M. Abramowitz and I. Stegun, Handbook of Mathematical Functions With Formulas, Graphs, and Mathematical Tables. Washington, DC: NBS, 1964.

[32] G. Hahn and S. Shapiro, Statistical Models in Engineering. Hoboken, NJ: Wiley, 1994.

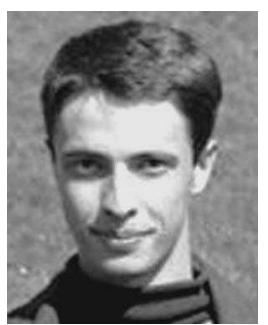

Lionel Bombrun (S'06-M'09) was born in Tournon, France, in 1982. He received the M.S and Ph.D. degrees in signal, image, speech, and telecommunications from the Grenoble National Polytechnic Institute (INPG), Grenoble, France, in 2005 and 2008, respectively.

In 2008, he was a Teaching Assistant at Phelma, Grenoble. Since October 2009, he has been a Postdoctoral Fellow with the French National Council for Scientific Research (CNRS) between the Grenoble Image sPeech Signal Automatics Laboratory (GIPSA-lab), Grenoble, and the Supelec, ONERA, NUS, DSTA Research Alliance (SONDRA), Gif-sur-Yvette, France. His research interests include signal and image processing, synthetic aperture radar remote sensing, polarimetry, and interferometry.

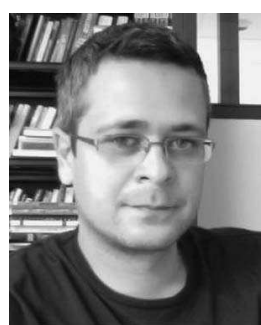

Gabriel Vasile (S'06-M'07) received the M.Eng. degree in electrical engineering and computer science and the M.S. degree in image, shapes, and artificial intelligence from the POLITEHNICA University, Bucharest, Romania, in 2003 and 2004, respectively. He received the Ph.D. degree in signal and image processing from Savoie University, Annecy, France, in 2007.

From 2007 to 2008, he was a Postdoctoral Fellow with the French Space Agency (CNES) and was with the French Aerospace Lab (ONERA), Palaiseau, France. Since 2008, he has been with the French National Council for Scientific Research (CNRS), where he is currently a Research Scientist and a member of the Grenoble Image sPeech Signal Automatics Laboratory (GIPSA-lab), Grenoble, France. His research interests include signal and image processing, synthetic aperture radar remote sensing, polarimetry, and interferometry.

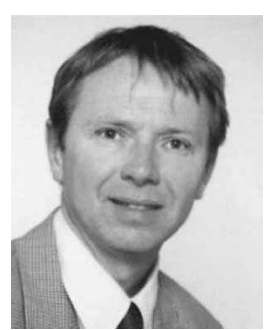

Michel Gay (M'10) received the B.Eng. degree in electrical engineering from the Institut des Sciences de l'Ingénieur de Montpellier, Montpellier, France, in 1987, and the Ph.D. degree in physics from the University Joseph Fourier, Grenoble, France, in 1999.

From 1988 to 2003, he was with Cemagref Grenoble, Grenoble, where he worked on electrical engineering for environmental applications. Since 2003, he has been a Research Engineer with the Grenoble Image sPeech Signal Automatics Laboratory (GIPSA-lab), Grenoble Institute of Technology, French National Council for Scientific Research (CNRS), Grenoble, France. His research interests include remote sensing, image processing, and the survey of Alpine glaciers.

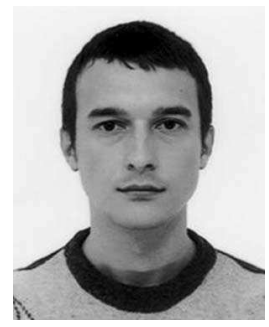

Felix Totir received the B.S. degree in radar and guidance systems from the Military Technical Academy of Bucharest, Bucharest, Romania, in 2002. He received the M.S. degree in telecommunications and the Ph.D. degree in signal processing from the University of Brest, Brest, France, in 2003 and 2006, respectively.

$\mathrm{He}$ is currently with the Grenoble Image sPeech Signal Automatics Laboratory (GIPSA-lab), Grenoble, France. His research interests include radar signal processing, automatic object recognition, time-frequency analysis, dynamical systems, and cellular automata. 